\title{
PREVENTION OF MOTHER-TO-CHILD TRANSMISSION OF HIV: THE GEORGIAN EXPERIENCE
}

\author{
Tengiz Tsertsvadze ${ }^{1,2}$, Tea Kakabadze ${ }^{1}$, Ketevan Shermadini ${ }^{1}$, Akaki Abutidze ${ }^{1}$, Marika Karchava ${ }^{1}$, \\ Nikoloz Chkhartishvili ${ }^{1}$, Nino Badridze ${ }^{1}$, Zaza Bokhua ${ }^{3}$, Tengiz Asatiani ${ }^{3}$ \\ ${ }^{1}$ Infectious Diseases, AIDS and Clinical Immunology Research Center, Tbilisi, Georgia \\ ${ }^{2}$ Iv. Javakhishvili Tbilisi State University Faculty of Medicine, Tbilisi, Georgia \\ ${ }^{3}$ Georgian Obstetrics and Gynecologists Association, Tbilisi, Georgia
}

\begin{abstract}
SUMMARY
Objective: The objective of this paper is to review experience in prevention of mother-to-child transmission (PMTCT) of HIV in Georgia.

Background: PMTCT is one of the strategic priorities in Georgia. The first case of HIV infection in pregnant women was reported in 1999. Starting 2005 the National Programme on PMTCT became operational.

Materials and Methods: One hundred sixteen HIV voluntary counselling and testing (VCT) centers operate throughout the country at antenatal clinics. According to the National PMTCT protocol, all first time attending pregnant women are offered Voluntary Counselling and Testing (VCT). Testing on HIVIAIDS is based on identification of HIV antibodies by screening method and all positive results are referred to the Infectious Diseases, AIDS and Clinical Immunology Research Center (IDACIRC) for the further investigation (confirmation by Western Blot assay) and further management. Data collection was made retrospectively, using information from IDACIRC National HIVIAIDS Data Base, VRF for the period 1999-2007.

Results: Prevalence of HIV among pregnant women availing VCT services in 2006 was $0.03 \%$. As of December, 2007 total 69 pregnancies of 64 women were registered at the IDACIRC.

Fifty eight women (90.6\%) acquired infection through heterosexual contact. None of the HIV positive women reported intravenous injection of illicit drugs. The majority of the HIV infected pregnant women had one sexual partner (90.6\%). Of children delivered by 51 positive partners $41(80 \%)$ were infected through injecting drugs intravenously and $10(20 \%)$ persons through heterosexual contacts.

Throughout the period 1999-2007 14 pregnant women received PMTCT services only partially. In 2 cases children were HIV-infected. In 12 pregnancies women received AZT in about the 28th week of pregnancy. No case of HIV transmission to child was recorded in this group. In 32 cases pregnant women received full prophylaxis therapy and all children were negative for HIV infection. Among 6 pregnant women admitted at IDACIRC later than the 28th week of pregnancy only 1 child was infected. As of December 2007, 5 women are still pregnant. Three of them receive antiretroviral drugs (ARV) prophylaxis with AZT+3TC+SQV/r. Two women are under 28 weeks of gestational age.

Conclusion: Over the last several years the national response to AIDS in Georgia achieved significant progress. The provision of comprehensive packages of PMTCT services in Georgia has been shown to minimize the risk of vertical transmission. As described above none of the women completing full course of ARV prophylaxis, combined with appropriate infant feeding, transmitted HIV to their children. PMTCT programmes are indisputably the main entry point not only for HIV related care and treatment for women, but also for other comprehensive care and prevention.
\end{abstract}

Key words: HIV, prevention of mother-to-child transmission, antiretroviral drugs, prophylaxis, Georgia

Address for correspondence: T. Tsertsvadze, Infectious Diseases, AIDS and Clinical, Immunology Research Center, 16 Al. Kazbegi Ave, Tbilisi 0160, Georgia. E-mail: tengizt@gol.ge, aids@gol.ge

\section{INTRODUCTION}

The HIV pandemic is one of the most serious health crises in human history. The Joint United Nations Programme on AIDS (UNAIDS) and the World Health Organization (WHO) estimate that approximately 33.2 million people were living with HIV worldwide in 2007, including 2.5 million children under 15. A disproportionate burden has been placed on women and children, who in many settings continue to experience high rates of new HIV infections and of HIV-related illnesses and death. In
2005 alone, an estimated 420,000 children were newly infected with HIV (1).

Georgia - the independent nation formerly part of the Soviet Union - is classified as having a low-prevalence HIV epidemic with an estimated prevalence of $0.09 \%$. The first case of HIV infection in Georgia was reported in 1989. As of December 2007, a total of 1,472 HIV/AIDS cases have been registered officially in Georgia, including 38 children under 15 . The estimated number of HIV/AIDS cases is 3,000. 
Similarly to other Newly Independent States (NIS) of the Former Soviet Union (FSU), Georgia's HIV epidemic is strongly associated with injection drug use (IDU) accounting for approximately $60 \%$ of all reported cases. However over the last several years heterosexual route is gaining on importance, with nearly $33 \%$ of cases attributed to heterosexual transmission. At the same time, annual number of newly reported HIV infections among females has risen each year and the number has almost tripled over the last three years (2005-2007) (Fig. 1).

Despite HIV low prevalence, Georgia is believed to be at risk for an imminent epidemic spread of HIV due to widespread injecting drug use, high rates of sexually transmitted infections (STI) and by hepatitis $\mathrm{B}$ and $\mathrm{C}$, which share common routes of transmission and by intense population movement between neighboring high-prevalence countries such as Ukraine and Russia.

Georgia was one of the countries in the NIS that immediately reacted to the epidemic in early 1990s. National efforts have led to a number of key achievements: establishment of HIV/AIDS service organizational structures, development of legal, policy and programme friendly environment. There is mandatory notification of HIV infection. All identified cases are referred to the Infectious Diseases, AIDS and Clinical Immunology Research Center (IDACIRC) for HIV-related care. Diagnostics and treatment of AIDS patients are provided at the highest attainable standards. The treatment services are provided at this Center and two regional treatment facilities coordinated by IDACIRC.

Since 2004 Georgia has demonstrated substantial progress in addressing HIV epidemic, especially in the scaling-up Antiretroviral Therapy (ART) as result of strengthened resource allocation from the Global Fund to fight AIDS, Tuberculosis and Malaria (GFATM). Through financial support of GFATM and under coordination of IDACIRC, Georgia first among NIS ensured universal access to ART, which along with provision of antiretroviral drugs (ARV) also implies comprehensive clinical and laboratory care of all People Living with HIV/AIDS (PLHA).

Mother-to-child transmission (MTCT) of HIV remains a major public health problem worldwide, especially in developing countries. Prevention of mother-to-child transmission (PMTCT) of HIV is one of the major strategic priorities as defined by the National Strategic Plan of Action (NSPA) of Georgia. HIV can be transmitted from an infected mother to her child in utero, during

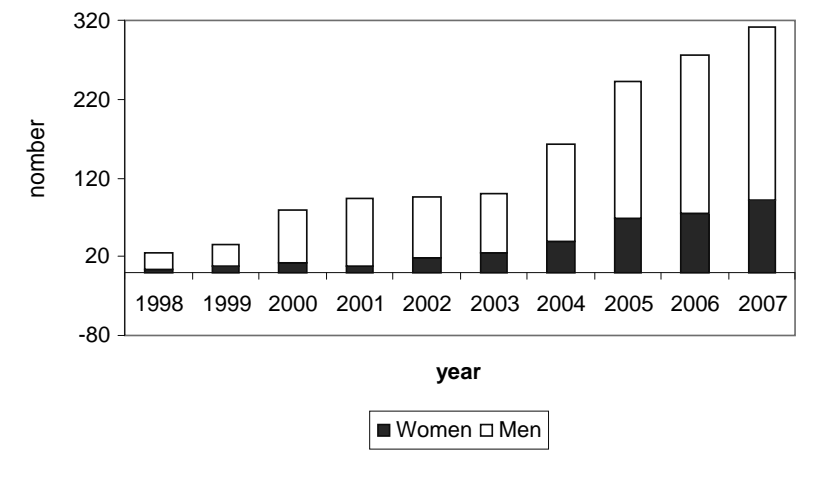

Fig. 1. Gender distribution of newly diagnosed HIV cases by years. delivery, and postnatally through breastfeeding. Implementation of effective PMTCT strategies in developed countries reduced transmission rates to less than $2 \%$, while the rate of new infections in children remains high in developing countries (2-5).

In line with Universal Access initiative, Georgia has launched National Programme on PMTCT in 2005. Before the integration of PMTCT into existing routine antenatal health services, access to HIV Voluntary Counselling and Testing (VCT) component of the PMTCT was limited to capital city of Tbilisi, where the VCT was provided from 2002 to 2005 by the Elizabeth Glaser Pediatric AIDS Foundation (EGPAF) PMTCT project operated by the Maternal and Child Care Union.

National Programme on PMTCT is based on the national PMTCT policy and action plan developed in 2003-2005 with the support of UNICEF. The Program envisages:

- primary prevention of HIV among women of reproductive age;

- reducing unintended pregnancies among women with HIV;

- reducing transmission from HIV-positive mother to her child;

- providing care, treatment and support to mothers and their infants and families.

PMTCT services are provided free of charge and are supported by the Government of Georgia, Vishnevskaya-Rostropovich Foundation (VRF) and GFATM. In general all pregnant women with confirmed HIV diagnosis are involved in the PMTCT programme funded by the GFATM and being implemented by the IDACIRC in partnership with Georgian Obstetricians and Gynecologists Association (GOGA). IDACIRC has an experience of providing PMTCT services to HIV-infected women since 1999, when the first case of HIV was detected in pregnant woman. The comprehensive approach to reducing mother-to-child transmission is based on existing evidence (6-12) and includes: provision of prophylactic ART; counselling and support for delivery and infant feeding; HIV-related care and ART of mother and infant, including cotrimoxazole prophylaxis.

\section{MATERIALS AND METHODS}

There are 116 VCT Centers for pregnant women in Georgia. VCT centers distribution is the following: 19 of them are located at Women Health Centers, 88 in maternity hospitals, and other 9 VCT centers are allocated in different regional hospitals of Georgia.

\section{Voluntary Counselling and Testing}

To insure high quality VCT service availability, "Global Fund to Fight HIV/AIDS, TB and Malaria” (GFATM), IDACIRC together with Georgian Obstetric and Gynecologist Association (GOGA) trained VCT specialists for Maternity houses and WHC's. Training materials were developed in Georgian language and based on WHO recommendations.

According to the National PMTCT protocol, all first time attending pregnant women were offered VCT. VCT was provided according to the WHO recommendations. One to one as well as couple pre-test counselling included identification of risk behavioural factors, knowledge on HIV/AIDS, importance of HIV testing and interpretation of test results, prevention interventions etc. 
Testing on HIV/AIDS was based on identification of HIV antibodies by screening method, using high sensitive and specific rapid/simple tests. Detection of HIV antibodies was performed using Rapid Anti-HIV (1\&2) Test InTec PRODUCTS, INC. By protocol, all reactive samples were sent for confirmation (by Western Blot method) to IDACIRC.

Confirmation of HIV test results was done by retesting of sample using Vironostica HIV Uni-Form II Ag/Ab. bioMerieux, The Netherlands by ELISA with further confirmation by Western Blot method using HIV BLOT 2.2 Western Blot Assay.

After confirmation, results were sent back to the counsellors for post-test consultation. Post-test counselling was held in the same institution. All HIV-positive women were referred to IDACIRC for follow-up, psychological support and clinical/prophylactic interventions.

\section{ARV Prophylaxis}

Management of HIV-positive pregnant women was provided according to the national PMTCT guideline. According to guideline, PMTCT was initiated from the 28th week of gestation for all HIV-positive ARV naive pregnant women (without clinical and laboratory indications for HAART). ARV regimens were the following: 1. monotherapy with AZT or 2. combination of AZT+3TC, or 3. combination of AZT+ 3TC+ SQV/rtv. $\mathrm{AZT}+3 \mathrm{TC}+\mathrm{SQV} / \mathrm{rtv}$ - based drug regimen was considered as preferable and cost-effective regiment.

In case of AZT monotherapy or delayed diagnosis of HIV, NVP tablet was administered 2-3 hours before the delivery. Caesarean section was performed at the 38th week of gestation. I.v. AZT was administered during delivery. After delivery ARV treatment was stopped.

In case pregnant women is HIV-positive and ARV treatment [symptomatic HIV infection with relevant symptoms, lower CD4 count (CD4 count $<200 / \mathrm{mm}^{3}$ ) or CD4 is $201-350 / \mathrm{mm}^{3}$ and viral load is more than 100,000 copies/ml] was introduced in the 2 nd trimester of pregnancy, EFV was excluded from combination. If pregnant women already were on ART, EFV was switched to NVP.

All newborns born by HIV-positive mothers were given appropriate ARV treatment consisting of AZT+NVP. Dosage and prophylaxis duration in newborns were defined according to the national and WHO guidelines. All HIV-positive mothers were recommended not to breast-feed infant despite the ARV regimen.

In adition, PMTCT programme considered also cotrimoxazole PCP prophylaxis for children of HIV-positive mothers.

\section{Infants HIV Status Detection}

All infants, who were born from HIV positive mothers, were screened for HIV infection twice using virological test (HIV DNA PCR) at 48 hours and 4 months of age. Blood samples from the umbilical cord were not used for diagnostic evaluations because of potential contamination with maternal blood. Blood samples collected at the maternity house by the skilled professional were immediately delivered to the IDACIRC for investigation.

Till 2006, HIV-1 RNA in plasma was measured by commercially available quantitative Polymerase Chain Reaction (PCR) method (AMPLICOR HIV-1 MONITOR Test, version 1.5, Hoffmann-La Roche, Inc.) and run according to the protocol of the manufacturer. Since 2006 The COBAS TaqMan HIV-1 test (real time PCR) was used for quantification of HIV-1 RNA in hunam EDTA plasma, using the High Pure System Viral Nucleic Acid Kit for manual specimen preparation and the COBAS TaqMan 48 Analyzer for automated amplification and detection. The test can quantify HIV-1 RNA over range of 40-10.000,000 copies/ml.

A positive virological test (usually DNA) at 48 hours of age presumably meant that the infant was "probably HIV-infected" and was retested again with virological test using separate specimen for confirmation as soon as possible. Only the second HIV positive test can confirm infant's HIV infection. A negative result at this stage suggested an undetermined status.

A child with positive virological test result at the 4th month of age was retested again using separate specimen for confirmation in order to indicate whether the infant was HIV-infected and should be clinically evaluated to develop a management strategy. Negative HIV test result pointed to HIV-negative status.

\section{Data Collection}

Data collection was made retrospectively, using information from IDACIRC National HIV/AIDS Data Base, VRF for the period 1999-2007.

Medical charts and case investigation records were reviewed for HIV-positive women (age, educational level, employment, marital status, risk behavior, transmission mode, clinical and laboratory markers).

\section{RESULTS}

To facilitate full-scale implementation of the PMTCT programme IDACIRC and GOGA trained 670 professionals working in antenatal health services in 2004-2005 to ensure high quality VCT services at the antenatal clinics throughout the country.

In 2006, 42,430 pregnant women agreed and received VCT services (total number of deliveries in 2006: 48,181). Fourteen women were found to be HIV-positive, thus the prevalence of HIV among pregnant women availing VCT services was $0.03 \%$.

A total of 69 pregnancies in $64 \mathrm{HIV}$-infected women were reported in Georgia from 1999 to 2007. Women were identified through HIV case investigation, EGPAF project and National Programme on PMTCT.

Majority of women were falling into the age group of 20 to 30 at the time of HIV diagnosis [mean age 27.3 (standard deviation 6.4; range 17-49)], had a high school level of education (62.5\%), were unemployed (76.6\%), and were married (82.8) (Table 1). Nine women were internally displaced persons.

Distribution of HIV positive pregnant women by regions of Georgia correlated with HIV prevalence in these regions, with most of cases reported from the capital city of Tbilisi (Fig. 2).

Fifty eight women (90.6\%) acquired infection through heterosexual contact, in 6 cases $(9.4 \%)$ mode of transmission was not ascertained. None of the HIV-positive women reported intravenous injection of illicit drugs. The majority of the HIVinfected pregnant women had one sexual partner (90.6). There was low demand and use of condoms and contraceptives in this population. With regard to sexual partners, 51 (69.7\%) partners were HIV-positive, 6 (9.4\%) were HIV-negative, in 7 (10.9\%) cases partner HIV status was not detected. Among 51 positive partners 41 (80\%) were infected through injecting 
Table 1. Demographic characteristics of 64 HIV-positive pregnant women, 1999-2007

\begin{tabular}{|c|c|}
\hline & No (\%) \\
\hline \multicolumn{2}{|l|}{ Age (years) } \\
\hline $17-19$ & $4(6.3)$ \\
\hline $20-24$ & 20 (31.2) \\
\hline $25-29$ & $22(34.4)$ \\
\hline $30+$ & 18 (28.1) \\
\hline \multicolumn{2}{|l|}{ Education } \\
\hline School level & $40(62.5)$ \\
\hline Higher level & $24(37.5)$ \\
\hline \multicolumn{2}{|l|}{ Employment } \\
\hline Unemployed & $49(76.6)$ \\
\hline Employed & 12 (18.8) \\
\hline Student & $3(4.7)$ \\
\hline \multicolumn{2}{|l|}{ Marital status } \\
\hline Married & 53 (82.8) \\
\hline Divorced & $3(4.7)$ \\
\hline Single & $5(7.8)$ \\
\hline Widowed & $3(4.7)$ \\
\hline
\end{tabular}

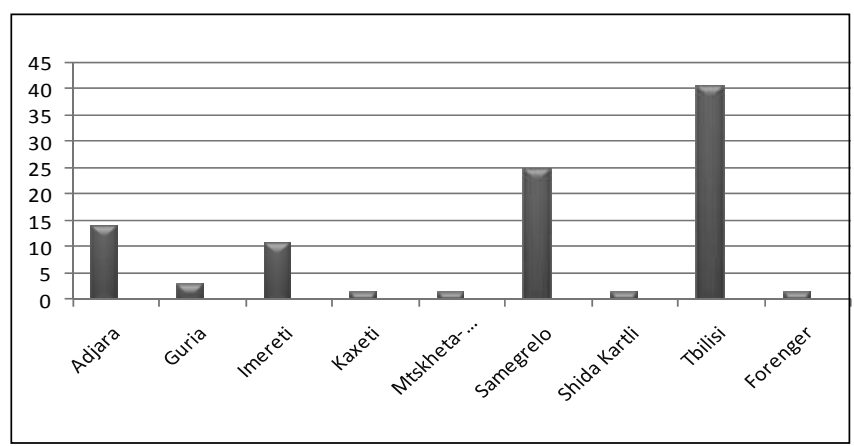

Fig. 2. Distribution of HIV-infected pregnant women by regions of Georgia.

drugs intravenously and 10 (20\%) persons through heterosexual contacts (Table 2).

All HIV-positive pregnant women admitted at the IDACIRC underwent the comprehensive clinical and laboratory investigations. CD4+ cell count in 36 cases of pregnancy was more then 500 cells $/ \mathrm{mm}^{3}$, in 27 cases $200-500$ cells $/ \mathrm{mm}^{3}$, and 6 cases were below 200 cells $/ \mathrm{mm}^{3}$. AIDS was determined in 6 cases of pregnancy. Viral load was above 1,000 copies/ml in 62 cases and below this level in 7 cases. Viral load did not change the PMTCT design. Anemia was frequent problem in HIV-positive pregnant women in Georgia. Main reason of anemia was iron deficiency. Chronic hepatitis $C$ and/or B co-infection was revealed in 6 cases of pregnancy. Among them 4 were co-infected with hepatitis $C$ and two were both HBsAg and anti-HCV-positive. Co-infection with lung tuberculosis was detected in 2 cases. None of the patients was infected with other sexually transmitted (syphilis and/or gonorrhea) disease (Table 3).

Throughout the period 1999-2007 prophylactic strategy in HIV-positive pregnant women was tailored individually according to the gestation age, HIV disease stage, availability of ARVs, patient's desire to receive prophylaxis etc.

14 pregnant women received PMTCT services only partially. The reason was: late HIV diagnosis, limited access to ARV (from 1999 till 2004); refusal by pregnant women. In 2 cases children
Table 2. Risk factors among 64 HIV-positive women

\begin{tabular}{|l|l|}
\hline & No (\%) \\
\hline $\begin{array}{l}\text { Number of sexual partners } \\
\text { One sexual marital/non-marital partner } \\
\text { Multiple sexual partners }\end{array}$ & $\begin{array}{l}58(90.6) \\
6(9.4)\end{array}$ \\
\hline Condoms / Contraceptive methods using & $9(14.0)$ \\
\hline Injection drug use & $0(0)$ \\
\hline $\begin{array}{c}\text { Sexual partner HIV status } \\
\text { Positive }\end{array}$ & $51(69.7)$ \\
Negative & $6(9.4)$ \\
Unknown & $7(10.9)$ \\
\hline Mode of transmission in sexual partners & \\
Injection drug use & $41(80.4)$ \\
Heterosexual contact & $10(19.6)$ \\
\hline
\end{tabular}

Table 3. Results of laboratory investigation of HIV-positive pregnant women

\begin{tabular}{|l|l|}
\hline Laboratory tests and co-infections & No. (\%) \\
\hline $\begin{array}{l}\text { CD4 count, cells/mm }{ }^{3} \\
>500\end{array}$ & $36(52.2)$ \\
$200-500$ & $27(39.1)$ \\
$<200$ & $6(8.7)$ \\
\hline Viral load & \\
$>1,000$ & $7(10.1)$ \\
$<1,000$ & $62(89.9)$ \\
\hline Hemoglobin & \\
$>100$ g/l & $50(72.4)$ \\
$<100$ and $>80$ g/l & $18(26.0)$ \\
$<80$ g/l & $1(1.5)$ \\
\hline Co-infections & \\
Chronic hepatitis C & $4(5.8)$ \\
Chronic hepatitis B and C & $2(2.9)$ \\
Tuberculosis & $2(2.9)$ \\
Syphilis, gonorrhea & $0(0)$ \\
\hline
\end{tabular}

were HIV-infected. Both cases were registered before 2004. In both cases no ARV was used, delivery was performed vaginally and children received infant feeding. In addition, in one case HIV status of the child could not be determined due to loss of follow-up.

In case of other 12 pregnancies women received AZT in about the 28th week of pregnancy. During delivery they received one tablet of NVP or i.v. AZT. Caesarean section was performed in all cases. Newborns received NVP in combination with AZT. They were fed by infant formula or breast. No case of HIV transmission to child was recorded in this group.

In 32 cases pregnant women received full prophylactic therapy and all children were negative for HIV infection. Among this group 2 women refused caesarean section.

Of 6 women admitted to IDACIRC after the 28th week of pregnancy only 1 child was born infected. In this case pregnant woman was admitted in the 3rd trimester of pregnancy with the late stage of HIV infection (Table 4).

As of December 2007, 5 women are still pregnant. Three of them receive ARV prophylaxis with AZT+3TC+SQV/rtv. Two women are under 28 weeks of gestational age. 
Table 4. PMTCT in Georgia

\begin{tabular}{|c|c|c|c|c|c|c|c|c|}
\hline \multirow{3}{*}{$\begin{array}{l}\text { No. of } \\
\text { cases }\end{array}$} & \multicolumn{3}{|c|}{ Antiretroviral treatment (ART) } & \multirow[b]{3}{*}{$\begin{array}{c}\text { Mode } \\
\text { of delivery }\end{array}$} & \multirow[b]{3}{*}{$\begin{array}{c}\text { ART } \\
\text { of infant }\end{array}$} & \multirow[b]{3}{*}{$\begin{array}{l}\text { Infant } \\
\text { feeding }\end{array}$} & \multirow[b]{3}{*}{$\begin{array}{l}\text { No. of HIV- } \\
\text { infected } \\
\text { children }\end{array}$} & \multirow[b]{3}{*}{ Comments } \\
\hline & \multicolumn{3}{|c|}{ During pregnancy } & & & & & \\
\hline & $\begin{array}{l}\text { Before/From } \\
\text { 28th week of } \\
\text { gestation }\end{array}$ & $\begin{array}{l}\text { Later than 28th } \\
\text { week of gestation }\end{array}$ & During delivery & & & & & \\
\hline 14 & -- & -- & -- & $\begin{array}{l}\text { caesarean } \\
\text { section (11) } \\
\text { vaginal } \\
\text { delivery (3) }\end{array}$ & $\begin{array}{l}\text { NVP (4) or } \\
\text { AZT+NVP } \\
\text { (7) or } \\
\text { AZT+3TC } \\
\quad(1) \text { or } \\
\text { no ARV (2) }\end{array}$ & $\begin{array}{l}\text { infant formula } \\
\text { (10) or breast } \\
\text { feeding (4) }\end{array}$ & 2 & \\
\hline 12 & AZT & -- & $\begin{array}{l}\text { NVP (12) or/and } \\
\text { i.v. AZT (10) }\end{array}$ & $\begin{array}{l}\text { caesarean } \\
\text { section }\end{array}$ & $\mathrm{NVP}+\mathrm{AZT}$ & $\begin{array}{l}\text { infant formula } \\
\text { (10) or breast } \\
\text { feeding (2) }\end{array}$ & 0 & $\begin{array}{l}\text { there was limited } \\
\text { access to ARVs in } \\
\text { the country }\end{array}$ \\
\hline 32 & $\begin{array}{c}A Z T+3 T C+S Q V / \\
\text { rtv }\end{array}$ & -- & i.v. AZT & $\begin{array}{l}\text { caesarean } \\
\text { section (30) } \\
\text { or vaginal } \\
\text { delivery (2) }\end{array}$ & $A Z T+N V P$ & infant formula & 0 & $\begin{array}{l}2 \text { women refused } \\
\text { caesarean section }\end{array}$ \\
\hline 6 & - & $A Z T+3 T C+S Q V / r t v$ & $\begin{array}{l}\text { i.v. AZT } \\
+ \text { NVP }\end{array}$ & $\begin{array}{l}\text { caesarean } \\
\text { section }\end{array}$ & $A Z T+N V P$ & infant formula & 1 & $\begin{array}{l}\text { admission in the } \\
\text { late stage } \\
\text { of pregnancy }\end{array}$ \\
\hline
\end{tabular}

Note: Caesar"ean section in all cases were planned and performed at 38th week of gestation except of cases when obstetricians assessed danger of premature delivery. In these cases section was done 1-2 weeks before planned data

Total number of HIV-exposed newborns was 58 from 57 deliveries. In one case twins were born. Totally 46 children were HIV-negative. 3 children were identified as HIV-positive. One child died before HIV investigation. Status of one child is undetermined because of lost of follow-up. HIV status of 7 children is under investigation (Table 5).

\section{DISCUSSION}

Over the last several years the national response to AIDS in Georgia attracted significant attention. Implementation of na-

Table 5. Outcomes of delivery among HIV-infected pregnant women in Georgia

\begin{tabular}{|l|l|}
\hline Outcomes of pregnancy & No. (\%) \\
\hline Total number of HIV exposed newborns & $58(100)$ \\
HIV-positive & $3(5.2)$ \\
HIV-negative & $46(79.4)$ \\
Unknown & $7(12.1)$ \\
Under investigation & $1(1.7)$ \\
Lost of follow-up & $1(1.7)$ \\
Death before HIV investigation & $1(1.7)$ \\
\hline Death in infancy & $6(10.3)$ \\
\hline Premature delivery (before 37 weeks of gestation) & $8(13.8)$ \\
\hline Low birth weight (<2,500 g) & \\
\hline
\end{tabular}

tionwide programme on PMTCT should be regarded as another major step towards universal access universal access of to HIV prevention, treatment, care and support along with the achievement of universal coverage with ART. All services are implemented in compliance with universal access of basic principles, implying that services have to be equitable, accessible, affordable, comprehensive and sustainable over the long-term (13).

Provision of comprehensive packages of PMTCT services in Georgia has been shown to minimize the risk of vertical transmission. As described above none of the women completing full course of ARV prophylaxis, combined with appropriate infant feeding, transmitted HIV to their children.

PMTCT programmes are indisputably the main entry point not only for HIV-related care and treatment for women, but also for other comprehensive care and prevention.

Although most of the pregnant women were infected through heterosexual contacts, majority of them acquired infection from their IDU partner. In Eastern European countries, with more mature epidemics and high prevalence in injecting drug users, sexual transmission from users to non-injecting sexual partners has long been the dominant mode of heterosexual transmission (14). This interpretation is very important as it confirms that as in early years, Georgia's HIV epidemic was driven by IDU $(15,16)$. At the same time, female sexual partners of IDU may serve as bridging population for expansion of HIV epidemic. Implementation of universal access to PMTCT in Georgia along with preventive interventions targeting IDUs can serve as key element for halting spread of the infection from population at risk to general public and avoiding wide-scale epidemic in the country. 


\section{REFERENCES}

1. Joint United Nations Programme on HIV/AIDS (UNAIDS) and World Health Organization (WHO). AIDS epidemic update: December 2007. Geneva: UNAIDS/WHO; 2007.

2. Kourtis AP, Bulterys M, Nesheim SR, Lee FK. Understanding the timing of HIV transmission from mother to infant. JAMA. 2001 Feb 14;285(6):709-12.

3. European Collaborative Study. Mother-to-child transmission of HIV infection in the era of highly active antiretroviral therapy. Clin Infect Dis. 2005 Feb 1;40(3):458-65.

4. Fowler MG, Lampe MA, Jamieson DJ, Kourtis AP, Rogers MF. Reducing the risk of mother-to-child human immunodeficiency virus transmission: past successes, current progress and challenges, and future directions. Am J Obstet Gynecol. 2007 Sep;197(3 Suppl):S3-9.

5. De Cock KM, Fowler MG, Mercier E, de Vincenzi I, Saba J, Hoff E, et al. Prevention of mother-to-child HIV transmission in resource-poor countries: translating research into policy and practice. JAMA. $2000 \mathrm{Mar}$ 1;283(9):1175-82.

6. World Health Organization. Antiretroviral drugs for treating pregnant women and preventing HIV infection in infants: guidelines on care, treatment and support for women living with HIV/AIDS and their children in resource-constrained settings. Geneva: WHO; 2004.

7. Hawkins D, Blott M, Clayden P, de Ruiter A, Foster G, Gilling-Smith C, et al; BHIVA Guidelines Writing Committee. Guidelines for the management of HIV infection in pregnant women and the prevention of mother-to-child transmission of HIV. HIV Med. 2005 Jul;6 Suppl 2:107-48.

8. U.S. Department of Health and Human Services. Public Health Service Task Force recommendations for use of antiretroviral drugs in pregnant HIV-1-infected women for maternal health and interventions to reduce perinatal HIV-1 transmission in the United States. Rockville: DHHS; 2005
9. Cooper ER, Charurat M, Mofenson L, Hanson IC, Pitt J, Diaz C, et al; Women and Infants' Transmission Study Group. Combination antiretroviral strategies for the treatment of pregnant HIV-1-infected women and prevention of perinatal HIV-1 transmission. J Acquir Immune Defic Sydnr. 2002 Apr 15;29(5):484-94.

10. International Perinatal HIV Group. The mode of delivery and the risk of vertical transmission of human immunodeficiency virus type 1 : a meta-analysis of 15 prospective cohort studies. N Engl J Med. 1999 Apr 1;340(13):977-87.

11. The Breastfeeding and HIV International Transmission Study (BHITS) Group. Late postnatal transmission of HIV-1 in breast-fed children: an individual patient data meta-analysis. J Infect Dis. 2004 Jun 15;189(12):2154-66.

12. Taha TE, Hoover DR, Kumwenda NI, Fiscus SA, Kafulafula G, Nkhoma $\mathrm{C}$, et al. Late postnatal transmission of HIV-1 and associated factors. J Infect Dis. 2007 Jul 1;196(1):10-4

13. Joint United Nations Programme on HIV/AIDS [homepage on the Internet]. Geneva: UNAIDS. Towards universal access: scaling up HIV prevention, treatment, care and support [cited 2008 Feb 15]. Available from: http://data.unaids.org/pub/InformationNote/2006/20060324_HLM_ GA_A60737_en.pdf.

14. Donoghoe MC, Matic S. HIV-1 in eastern Europe. Lancet. 2003 May 31;361(9372):1910-1.

15. Tkeshelashvili-Kessler A, del Rio C, Nelson K, Tsertsvadze T. The emerging HIV/AIDS epidemic in Georgia. Int J STD AIDS. 2005 Jan;16(1): 61-7.

16. Stvilia K, Tsertsvadze T, Sharvadze L, Aladashvili M, del Rio C, Kuniholm $\mathrm{MH}$, et al. Prevalence of hepatitis C, HIV, and risk behaviors for blood-borne infections: a population-based survey of the adult population of T’bilisi, Republic of Georgia. J Urban Health. 2006 Mar;83(2):28998.

Received February 15, 2008 Accepted in revised form May 20, 2008 